\title{
Waves excited at a free surface in a half-infinite isotropic nonlinear elasic medium
}

\author{
Takao Momoi \\ 2-25-3 Shakujiidai, Nerimaku, Tokyo 177-0045, Japan \\ (Received August 3, 1999; Revised March 30, 2000; Accepted March 31, 2000)
}

\begin{abstract}
In nonlinear elastic equations, there exist two kinds of simple waves, i.e., Non-Coupled and Coupled Simple Waves which are alias named nonlinear $P$ and $S$ waves, respectively. Nonlinear equation used in this paper is of second order with respect to displacement and, as the result, used stress is also of second order. A used model is a two-dimensional one. The used stress condition is truely nonlinear instead of quasi-linear, where the latter is usually solved by use of perturbation procedure. In the model of a half-infinite elastic medium, the nonlinear stress-free surface condition is, as the first approximation, separated into three branches of stress conditions in the case of a direct-hit earthquake. For the incidence of Simple (nonlinear) Waves on the free surface, the reflected waves are evaluated for the above three branches of condition. The energy expression is then obtained in a weakly nonlinear case (the elastic constants associated with higher order terms are negligibly small). Among three branches of condition, the first one is a condition which causes ordinary reflected waves similar to $P$ and $S$ waves in a linear theory. The other two are conditions which produce retarded reflected waves with slow velocity near the free surface. As the result of the retardation, high energy flux occurs along the free surface. This high energy causes a large disaster on the occasion of large earthquake. When nonlinear $P$ wave is retarded near the free surface, Rayleigh-type nonlinear wave appears along the free surface. The wave is then propagated at a velocity a little less than that of linear $S$ wave. This behavior indicates the generation process of Rayleigh wave. These results are obtained by use of truely nonlinear stress condition at the free surface instead of quasi-linear one based on perturbation procedure.
\end{abstract}

\section{Introduction}

Various kinds of studies concerning nonlinear waves in solids have been carried out in nonlinear field. The works done in this field are summarized and discussed elaborately in the volumes edited by Jeffrey and Engelbrecht (1994) and Engelbrecht (1997).

In the model with a boundary surface, the nonlinear reflection from there will cause phase shifts resulting in possible narrowing or broadening of the pulse (Engelbrecht, 1983). In the similar manner, the transmitted wave is also affected (Litvin and Tsvankin, 1987).

In the case of a stress-free boundary surface, nonlinear Rayleigh waves are discussed by so many researchers, for example, Taniuti and Nishihara (1977), Jeffrey and Kawahara (1982), Parker and Talbot (1983), and Engelbrecht et al. (1988) among others. Much of them are treated in the quasilinear case by using perturbation procedures and so on. In this paper, the generation possibility of nonlinear Rayleigh waves is examined in the case of truely nonlinear surface condition.

Other than these studies, the biggest concern in the present study is the generation of the excited waves at the free surface in a nonlinear elastic medium.

In the present paper, the surface stress condition is treated in a truely nonlinear form instead of quasi-linear one (perturbation procedure). Nonlinear stress conditions are, in this

Copy right (C) The Society of Geomagnetism and Earth, Planetary and Space Sciences (SGEPSS); The Seismological Society of Japan; The Volcanological Society of Japan; The Geodetic Society of Japan; The Japanese Society for Planetary Sciences. paper, decomposed by use of a characteristic of direct-hit earthquake. We find then slow velocity solution near the free surface. Such slow velocity causes high energy concentration near the free surface.

In the previous papers (Momoi, 1990 and 1992; these papers will be referred to papers $\mathrm{A}$ and $\mathrm{B}$, respectively), we described two kinds of Simple Waves in a nonlinear elastic medium, that is to say, Non-Coupled Simple Wave (only a longitudinal component) and Coupled Simple Wave (a transverse component accompanied by a weak longitudinal component).

In a paper entitled "Simple Waves characterizing wave propagation in a nonlinear elastic medium" (Momoi, 1999; this paper will be referred to paper C), we have found that these Simple Waves are dominant waves in the field of a nonlinear elastic medium. It is then found that, as the first approximation, even complex waves can be analyzed as composite waves consisting of several fragments of Simple Waves. Using this concept, we shall develop the theory in this paper.

In this paper, using these Simple Waves as incident and reflected waves in place of consecutive nonlinear waves, we shall analyze the response of nonlinear waves to the truely nonlinear boundary conditions at the free surface in a halfinfinite isotropic elastic medium. This point is decisively different from other works cited in the foregoing parts. In this study, the problem is limited to the two-dimensional case.

In the development of the present theory, a dissipation term is not contained in the governing equation. The existence of that term in the nonlinear equation makes the problem so complicated that the solution of the equation cannot be 
obtained so easily. Therefore, the mathematical treatment concerning dissipation was not tried in this paper. That will be a remained problem in the future. However, dissipative effects are still dominant. Most of energy excited near the free surface is dissipated as energy of destruction near the free surface.

\section{Used Model}

As shown in Fig. 1, we shall use a two-dimensional model. $x$ and $z$ are the components of the Cartesian coordinates, where $x$ - and $z$-axes are rightward and downward positive, respectively. The free surface is situated along the $x$-axis. $u$ and $w$ are the displacement components in the direction of $x$ - and $z$-axes, respectively. In the later development of the theory, these $u$ and $w$ will be appropriately replaced by $u_{1}$ and $u_{3}$, respectively. In Fig. $1, \theta_{i}, \theta_{r N}$ and $\theta_{r C}$ indicate the incidence angle of the incident Simple Wave, the reflection angles of the reflected Non-Coupled and Coupled Simple Waves, respectively.

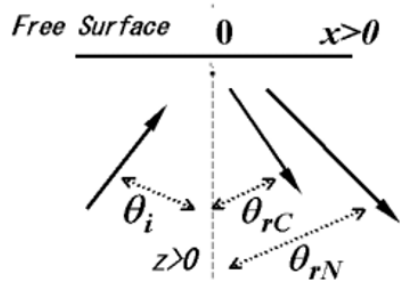

Fig. 1. Used model.

\section{Stress and Boundary Condition}

In a nondissipative thermoelastic isotropic solid medium, the energy function is expressed by three invariants associated with stress as follows (Bland, 1969).

$$
\begin{aligned}
E_{n}= & \lambda I_{1}^{2} / 2+\mu I_{2} \\
& +A I_{3} / 3+B I_{1} I_{2}+C I_{1}^{3} / 3,
\end{aligned}
$$

where $\lambda, \mu, A, B, C$ are elastic coefficients, $I_{1}, I_{2}, I_{3}$ are the tensor invariants which are expressed as

$$
\begin{aligned}
& I_{1}=U_{1}+U_{3}, \quad I_{2}=U_{1}^{2}+U_{3}^{2}+U_{5}^{2} / 2, \\
& I_{3}=U_{1}^{3}+U_{3}^{3}+\left(3 U_{1} U_{5}^{2}\right) / 4+\left(3 U_{3} U_{5}^{2}\right) / 4,
\end{aligned}
$$

with $U_{J}(J=1$ to 6$)$ being expressed

$$
\begin{aligned}
& U_{i j}=(1 / 2)\left(1+\delta_{i j}\right) U_{J}, \\
& U_{i j}=\left(u_{i j}+u_{j i}+u_{k i} u_{k j}\right) / 2,
\end{aligned}
$$

under Voigt Notation $\{i j=J\}$

$$
\{11=1,22=2,33=3,23=4,13=5,12=6\} \text {. }
$$

In $U_{i j}$ expression, duplicate $k$ in $u_{k i} u_{k j}$ indicates a summation over $k=1,2,3$ and

$$
\delta_{i j}: \text { Kronecker delta, } u_{i j}=\partial u_{i} / \partial x_{j} .
$$

The same development on energy function is done in the previous papers $\mathrm{A}$ and $\mathrm{B}$.
Elastic coefficients in expression (1a) will be used in a form normalized by $\mu$ as follows.

$$
\begin{aligned}
& L_{m}=\lambda / \mu, A_{m}=A / \mu, \\
& B_{m}=B / \mu, C_{m}=C / \mu .
\end{aligned}
$$

The stress tensor is related with energy function as follows.

$$
S_{i j}=\partial E_{n} / \partial u_{i j}
$$

Taking the terms up to second-order of $u$ and $w$, we have the following expressions in the two-dimensional case.

$$
\begin{aligned}
S_{11} / \mu= & g_{1} u_{x}^{2} / 2+f_{1} u_{z}^{2} / 2 \\
& +u_{x} v_{p}^{2} / v_{s}^{2}+f_{2} u_{z} w_{x}+f_{1} w_{x}^{2} / 2 \\
& +L_{m} w_{z}+g_{2} u_{x} w_{z}+g_{2} w_{z}^{2} / 2, \\
S_{33} / \mu= & L_{m} u_{x}+g_{2} u_{x}^{2} / 2 \\
& +f_{1} u_{z}^{2} / 2+f_{2} u_{z} w_{x}+f_{1} w_{x}^{2} / 2 \\
& +g_{2} u_{x} w_{z}+v_{p}^{2} w_{z} / v_{s}^{2}+g_{1} w_{z}^{2} / 2, \\
S_{13} / \mu= & u_{z}+f_{1} u_{x} u_{z}+w_{x}+f_{2} u_{x} w_{x} \\
& +f_{1} u_{z} w_{z}+f_{2} w_{x} w_{z}, \\
S_{31} / \mu= & u_{z}+f_{2} u_{x} u_{z}+w_{x}+f_{1} u_{x} w_{x} \\
& +f_{2} u_{z} w_{z}+f_{1} w_{x} w_{z},
\end{aligned}
$$

where

$$
\begin{aligned}
f_{1} & =2+A_{m} / 2+B_{m}+L_{m}, \\
f_{2} & =1+A_{m} / 2+B_{m}, \\
g_{1} & =6+2 A_{m}+6 B_{m}+2 C_{m}+3 L_{m}, \\
g_{2} & =2 B_{m}+2 C_{m}+L_{m}, \\
u_{x} & =\partial u / \partial x, \quad u_{z}=\partial u / \partial z, \\
w_{x} & =\partial w / \partial x, \quad w_{z}=\partial w / \partial z,
\end{aligned}
$$

and $v_{p}$ (later used) and $v_{s}$ are the velocity of linear $P$ and $S$ wave, respectively.

In the case of a weakly nonlinear elastic medium $\left(L_{m} \gg\right.$ $\left.A_{m}, B_{m}, C_{m}\right)$, the above expressions become

$$
\begin{aligned}
& f_{1}=2+L_{m}, \quad f_{2}=1, \\
& g_{1}=6+3 L_{m}, \quad g_{2}=L_{m},
\end{aligned}
$$

where weak nonlinearity signifies that third order terms are negligibly small in energy function (1a).

In practice, analytical reduction will be carried out later in the weakly nonlinear case described in (1e) in order to make easy the physical interpretation about the phenomenon of the wave propagation (for better visibility of physical feature).

The boundary conditions at the free surface are given by vanishing of normal and tangential stresses on that surface, that is,

$$
S_{33} / \mu=0, \quad S_{13} / \mu=0,
$$

where $\left\{S_{33} / \mu, S_{13} / \mu\right\}$ are given by (1d).

In this paper, the development of the theory will be made under a particular condition of a direct-hit earthquake. A direct-hit earthquake implies that the incidence angle of the incident wave is small, so that the wave front of the waves is 
considered to be nearly parallel to the free surface. Therefore, we can put, as the first approximation,

$$
u_{x}=0, \quad w_{x}=0,
$$

i.e., derivative of $u$ and $w$ with respect to $x$ is zero. Then (1f) is reduced to

$$
\begin{aligned}
& S_{13} / \mu=u_{z}\left(v_{s}^{2}+v_{p}^{2} w_{z}\right) / v_{s}^{2}=0, \\
& S_{33} / \mu=v_{p}^{2}\left(u_{z}^{2}+2 w_{z}+3 w_{z}^{2}\right) /\left(2 v_{s}^{2}\right)=0,
\end{aligned}
$$

where the coefficients (1e) are used which are associated with a weakly nonlinear elastic medium.

By solving the above two equations, these conditions are separated into the following three branches of boundary conditions.

$$
\begin{array}{ll}
\text { [I] } u_{z}=0, \quad w_{z}=0, \\
\text { [II } \quad u_{z}=0, w_{z}=-2 / 3, \\
\text { [III }] \quad u_{z}= \pm\left(2 v_{p}^{2}-3 v_{s}^{2}\right)^{1 / 2} v_{s} / v_{p}^{2}, \\
w_{z}=-\left(v_{s}^{2} / v_{p}^{2}\right),
\end{array}
$$

where $\{u, w\}$ indicates the total wave described in (2f) given later.

Among the above three pairs of conditions, the first condition [I] refers to that in the case of a linear elastic theory, while the last two [II] and [III] conditions are those that are produced from nonlinear terms of stress expressions. The latter two cause nonlinear effect on the reflected waves, particularly excitation of waves at the free surface.

\section{Simple Wave}

By use of the stress expressions described in (1d), fundamental equation is given by

$$
\rho \partial^{2} u_{i} / \partial t^{2}=\partial S_{i j} / \partial x_{j} \quad(i=1,3),
$$

where $\rho$ and $t$ are a density of a medium and a time variable, respectively.

These equations are expressed as, in one-dimensional case ( $x$ direction),

$$
\begin{aligned}
& u_{t 2}=u_{x 2} v_{p}^{2}+3 u_{x} u_{x 2} v_{p}^{2}+v_{p}^{2} w_{x} w_{x 2} \\
& w_{t 2}=u_{x 2} v_{p}^{2} w_{x}+u_{x} v_{p}^{2} w_{x 2}+v_{s}^{2} w_{x 2}
\end{aligned}
$$

where

$$
\begin{aligned}
& u_{t 2}=\partial^{2} u / \partial t^{2}, \quad w_{t 2}=\partial^{2} w / \partial t^{2}, \\
& u_{x 2}=\partial^{2} u / \partial x^{2}, w_{x 2}=\partial^{2} w / \partial x^{2}, \\
& u_{x}=\partial u / \partial x, \quad w_{x}=\partial w / \partial x, 0
\end{aligned}
$$

and the coefficients (1e) are used.

In order to further simplify the above equations, we shall use the coordinates $\left\{t_{r}, k_{r}\right\}$ moving at a velocity $v_{r}$, i.e., $\left\{t_{r}=\right.$ $\left.v_{r} t, k_{r}=v_{r} t-x\right\}$. This transformation is very familiar and frequenly used in the field of pure physics on nonlinear K-dV equation or Schrodinger equation (for example, Hazewinkel et al., 1995).

Following the above procedure, Eqs. (2a) are transformed and then the derivative term with respect to time $t_{r}$ is put to zero, since we are treating the wave propagation moving stationarily at a constant velocity $v_{r}$ in this paper. Furthermore, integrating these equations with respect to $k_{r}$, we have

$$
\begin{aligned}
& u_{k r} V_{p m}-3 u_{k r}^{2} v_{p}^{2} /\left(2 v_{s}^{2}\right)-v_{p}^{2} w_{k r}^{2} /\left(2 v_{s}^{2}\right)=0, \\
& -u_{k r} v_{p}^{2} w_{k r} / v_{s}^{2}+V_{s m} w_{k r}=0,
\end{aligned}
$$

where

$$
\begin{array}{ll}
V_{p m}=\left(v_{p}^{2}-v_{r}^{2}\right) / v_{s}^{2}, & V_{s m}=1-v_{r}^{2} / v_{s}^{2}, \\
u_{k r}=\partial u / \partial k_{r}, & w_{k r}=\partial w / \partial k_{r},
\end{array}
$$

Solving Eqs. (2b) in the case of a weakly nonlinear medium (using (1e)), we have

(i) Non-Coupled Simple Wave

$$
\begin{aligned}
& u=\left(2 k_{r 1} V_{p r} v_{s}^{2}\right) /\left(3 v_{p}^{2}\right), \quad w=0, \\
& V_{p r}=\left(v_{p}^{2}-v_{r 1}^{2}\right) / v_{s}^{2}, \quad k_{r 1}=v_{r 1} t-x,
\end{aligned}
$$

where $v_{r 1}$ is a velocity of Non-Coupled Simple Wave.

(ii) Coupled Simple Wave

$$
\begin{aligned}
& u=\left(k_{r 2} v_{s}^{2} V_{s r}\right) / v_{p}^{2}, \\
& w=s_{w}\left(k_{r 2} v_{s} W_{r 2}^{1 / 2}\right) / v_{p}^{2}, \\
& W_{r 2}=v_{s}^{2}\left(2+2 L_{m}-V_{s r}\right) V_{s r}, \\
& V_{s r}=1-v_{r 2}^{2} / v_{s}^{2}, \quad k_{r 2}=v_{r 2} t-x,
\end{aligned}
$$

where $v_{r 2}$ is a velocity of Coupled Simple Wave and $s_{w}$ is a double sign \pm associated with $w$.

Later, the displacement components $\{u, w\}$ used above will be appropriately replaced by the form $u=u_{e J}$ and $w=u_{f}$ with suffix $\{J=i N, i C, r N, r C\}$.

Simple Waves mentioned above are associated with the propagation along the $x$-axis. In practice, the incident or reflected waves are obliquely incident on or reflected from the free surface, so that the above Simple Waves must be converted into the expressions associated with the axis directed toward the propagation of incident or reflected waves, respectively.

\section{(a) Case of Reflected Non-Coupled Simple Wave}

In the coordinates after the clockwise rotation of the axes $\{x, z\}$ with angle $\pi / 2-\theta_{r N}$ (see Fig. 1), new axes $\left\{x_{e r N}, x_{f r N}\right\}$ are obtained, where the $x_{e r N}$-axis runs toward the propagation direction of reflected Non-Coupled Simple Wave. New expressions in the new coordinates are given by the replacements,

$$
x=x_{e r N}, z=x_{f r N}, u=u_{e r N}, w=u_{f r N}
$$

in the expression (2c), where $\left\{u_{e r N}, u_{f r N}\right\}$ are the displacement components of the reflected Non-Coupled Simple Wave associated with the coordinates $\left\{x_{e r N}, x_{f r N}\right\}$, respectively.

\section{(b) Case of Reflected Coupled Simple Wave}

In the coordinates after the clockwise rotation of the axes $\{x, z\}$ with angle $\pi / 2-\theta_{r C}$ (see Fig. 1), new axes $\left\{x_{e r C}, x_{f r C}\right\}$ are obtained, where the $x_{e r C}$-axis runs toward the propagation direction of reflected Coupled Simple Wave. New expressions in the new coordinates are given by the replacements,

$$
x=x_{e r C}, z=x_{f r C}, u=u_{e r C}, w=u_{f r C}
$$


in the expression (2d), where $\left\{u_{e r C}, u_{f r C}\right\}$ are the displacement components of the reflected Coupled Simple Wave associated with the coordinates $\left\{x_{e r C}, x_{f r C}\right\}$, respectively.

Suppose that $\left(u_{J}, w_{J}\right)$ are the displacement components of Non-Coupled $(J=r N)$ and Coupled $(J=r C)$ Simple Wave in the absolute coordinates $(x, z)$, respectively. $\left(u_{J}, w_{J}\right)$ are expressed as

$$
\begin{aligned}
& u_{J}=S_{J} u_{e J}-C_{J} u_{f J}, \\
& w_{J}=C_{J} u_{e J}+S_{J} u_{f J}, \\
& S_{J}=\sin \theta_{J}, C_{J}=\cos \theta_{J},(J=r N, r C)
\end{aligned}
$$

where the suffix $(e J, f J)$ implies that a letter $J$ is replaced by $r N$ or $r C$ like $(e r N, f r N)$ etc.

The total wave is expressed as, like that in a linear case (see later comment),

$$
\begin{aligned}
& u=u_{I}+u_{r C}+u_{r N}, \\
& w=w_{I}+w_{r C}+w_{r N} .
\end{aligned}
$$

In the above, $\left(u_{I}, w_{I}\right)$ are the displacement components of an incident Non-Coupled $(I=i N)$ or Coupled $(I=i C)$ Simple Wave, respectively, which will be given later. As for $u_{r N}, w_{r N}, u_{r C}$ and $w_{r C}$, these expressions are given by (2e).

Some mention is made here about the validity of the linear sum of incident, reflected Non-Coupled and Coupled Simple Waves as mentioned above. Since we are treating nonlinear waves, such a simple sum of each wave component is considered to be impossible, since the coupling terms are generated in the original nonlinear equation. In the case of Simple Wave, such a worry does not occur. The expressions of Simple Wave are linear combinations with respect to $t, x$ and $z$ (see (2c) and (2d)), while each term of the original nonlinear equation has a factor of second derivative with respect to $t, x$ and $z$, that is, $u_{t 2}, w_{t 2}, u_{x 2}$ and $w_{x 2}$ (see (2a)). These second derivatives become zero, so that both sides of original equations also become zero. Therefore, the expression (2f) of the total wave always satisfies the original nonlinear equation.

\section{Energy Flux Density of Reflected Wave}

Let $d E_{r n x}, d E_{r t x}, d E_{r n z}$ and $d E_{r t z}$ be the energy flux density of reflected waves (designated by suffix $r$ ) in the normal and transverse direction (suffix $n$ and $t$, respectively) on $x$ and $z$-plane (suffix $x$ and $z$, respectively). These energy densities are expressed as

$$
\begin{array}{ll}
d E_{r n x}=S_{11 r} u_{r t}, & d E_{r t x}=S_{31 r} w_{r t}, \\
d E_{r n z}=S_{33 r} w_{r t}, & d E_{r t z}=S_{13 r} u_{r t},
\end{array}
$$

where

$$
u_{r t}=\partial u_{r} / \partial t \text { and } w_{r t}=\partial w_{r} / \partial t
$$

with

$$
\begin{aligned}
u_{r}= & u_{r N}+u_{r C}, w_{r}=w_{r N}+w_{r C} \\
& \text { (total reflected wave) }
\end{aligned}
$$

and $\left\{S_{11 r}, S_{33 r}, S_{13 r}, S_{31 r}\right\}$ being stresses (1d) associated with the total reflected wave $(3 \mathrm{~b})$.

Let $d E_{r x}$ and $d E_{r z}$ be the total energy flux density of the reflected waves in the direction of $x$-and $z$-axes, respectively. These expressions are given by

$$
\begin{aligned}
& d E_{r x}=d E_{r n x}+d E_{r t x}, \\
& d E_{r z}=d E_{r n z}+d E_{r t z} .
\end{aligned}
$$

By use of the above expressions, we shall evaluate the energy flux density of the reflected waves in the following sections for each incidence of Non-Coupled or Coupled Simple Wave.

\section{Case of Incidence of Non-Coupled Simple Wave 6.1 Incident Non-Coupled Simple Wave}

In the coordinates after the counter-clockwise rotation of the axes $\{x, z\}$ with angle $-\pi / 2+d \theta_{i}$ (see Fig. 1), new axes $\left\{x_{e i N}, x_{f i N}\right\}$ are obtained, where the $x_{e i N}$-axis runs toward the propagation direction of incident Non-Coupled Simple Wave. In Fig. $1, \theta_{i}=\theta_{i N}$, where $\theta_{i N}$ is the incidence angle of incident Non-Coupled Simple Wave. New expressions in the new coordinates are given by the replacements,

$$
x=x_{e i N}, z=x_{f i N}, u=u_{e i N}, w=u_{f i N}
$$

in the expression (2c), where $\left\{u_{e i N}, u_{f i N}\right\}$ are the displacement components of incident Non-Coupled Simple Wave associated with the coordinates $\left\{x_{e i N}, x_{f i N}\right\}$, respectively. That is to say,

$$
\begin{aligned}
& u_{e i N}=2 V_{p i} v_{s}^{2}\left(v_{i N} t-x_{e i N}\right) /\left(3 v_{p}^{2}\right), \\
& u_{f i N}=0, \\
& V_{p i}=\left(-v_{i N}^{2}+v_{p}^{2}\right) / v_{s}^{2},
\end{aligned}
$$

where $v_{i N}$ is a velocity of the incident Non-Coupled Simple Wave.

Let $\left\{u_{i N}, w_{i N}\right\}$ be the displacement components of the incident Non-Coupled Simple Wave in the absolute coordinates. These are expressed as

$$
\begin{aligned}
& u_{i N}=S_{i N} u_{e i N}+C_{i N} u_{f i N}, \\
& w_{i N}=-C_{i N} u_{e i N}+S_{i N} u_{f i N}, \\
& S_{i N}=\sin \theta_{i N}, \quad C_{i N}=\cos \theta_{i N} .
\end{aligned}
$$

In the above, the incidence angle $\theta_{i N}$ is a small quantity, since we are treating the case of a direct-hit earthquake, i.e.,

$$
\theta_{i N}=d \theta_{i N} \cdot\left(d \theta_{i N}: \text { a small quantity }\right)
$$

\subsection{Procedure of analysis}

In solving equations in the case of linear boundary problem, we usually introduce unknown factors attached in product form in front of the terms indicating transmitted and reflected waves. In the case of nonlinear boundary problem, such unknown factors are not introduced, but the velocities of transmitted and reflected Simple Waves are used as unknown factors. These factors determine the gradients, i.e., amplitudes of the transmitted and reflected Simple Waves.

In order to solve equations, we require more condition to determine the reflection angle of the reflected waves. In the case of seismic wave, the wavelength is usually long, so that the wave front (the fragment of Simple Wave) has a long train along the boundary. Therefore, we shall apply Snell's Law though the problem is a nonlinear one. In the case of a half-infinite elastic medium, this law is given by

$$
v_{r 1} / \sin \theta_{r N}=v_{r 2} / \sin \theta_{r C}=v_{i} / \sin \theta_{i} .
$$

at the free surface $(\mathrm{z}=0)$, where $v_{i}, v_{r 1}$ and $v_{r 2}$ indicate the velocities of the incident Simple Wave, reflected Non-Coupled and Coupled Simple Waves, respectively. 
In this section, we are treating the case of the incidence of Non-Coupled Simple Wave, so that $v_{i}=v_{i N}$ and $\theta_{i}=\theta_{i N}$.

In the next section, the above relation will be used in the case of the incidence of Coupled Simple Wave with substitution of $v_{i}=v_{i C}$ and $\theta_{i}=\theta_{i C}$.

Now, the procedure of theoretical reduction will be described here. The reduction was carried out by use of a perturbation method.

Since we are treating the case of a direct-hit earthquake, $d \theta_{i N}$ is small. When Non-Coupled Simple Wave is in the interior of the elastic medium far away from the boundary, the wave is propagated at a velocity near that of linear $P$ wave, i.e., $v_{i N} \sim v_{p}$, as shown in the previous work (numerical result; Momoi, 1999). Therefore, $V_{p i}$ is a small value.

First, $d \theta_{i N}$ and $V_{p i}$ are put to zero and the solutions $\left\{v_{r 1}, v_{r 2}\right\}$ are obtained as the first approximation.

A set of simultaneous equations is then made up by using boundary conditions [I], [II] and [III] given in (1h) and Snell's law given in (5). Thereafter, these equations are solved with respect to $v_{r 1}$ and $v_{r 2}$, i.e., unknown velocities of the reflected Non-Coupled and Coupled Simple Waves.

As the second approximation, $\left\{v_{r 1}, v_{r 2}\right\}$ are obtained by introducing a perturbation terms $\left\{d v_{r 1}, d v_{r 2}\right\}$ and using the original boundary condition (1f). This original boundary condition then involves the terms associated with $\partial u / \partial x$ or $\partial w / \partial x(\neq 0)$. The solutions are obtained up to the terms of first or lowest (higher than first) order of $\left\{d \theta_{i N}, V_{p i}\right\}$.

Through the above-mentioned reduction, the nonlinear effect of the surface stress condition is wrapped in the velocity expressions $\left\{v_{r 1}, v_{r 2}\right\}$.

By using the velocities $v_{r 1}$ and $v_{r 2}$ obtained above, we can obtain the explicit expressions for Non-Coupled and Coupled reflected waves from $\{(2 \mathrm{c}),(2 \mathrm{~d})\}$. Furthermore, the explicit expressions for the components of energy flux density can be obtained from $(3 \mathrm{c})$.

The above-mentioned procedure of reduction was carried out by use of computer algebra. In order to make clear the physical interpretation, all the development of the theory was made in the case of weakly nonlinear medium, i.e., (1e).

Now the results of the reduction will be given in the following for each case associated with the boundary conditions [I], [II] and [III] in (1h).

\subsection{Reflected waves in the case of incidence of Non- Coupled Simple Wave}

The energy flux density of the incident Non-Coupled Simple Wave, $d E_{i N}$ is, first of all, evaluated.

$$
d E_{i N}=\left(4 V_{p i}^{2} v_{s}^{2} \mu\right) /\left(9 v_{p}\right)
$$

By using the above energy density, the energy flux density of reflected Simple Waves is normalized and the order will be evaluated.

Case [I], [II] and [III] in the following are the discussions associated with [I], [II] and [III] in boundary conditions (1h).

Case [I]

$$
\begin{aligned}
& v_{r 1}=v_{p}+d v_{r 1}, \quad v_{r 2}=v_{s}-d v_{r 2}, \\
& d v_{r 1}=V_{p i} v_{s}^{2} /\left(2 v_{p}\right), \quad d v_{r 2}=0, \\
& d E_{r x} / d E_{i N}=0, \\
& d E_{r z} / d E_{i N}=-1, \quad(\text { note suffix } z) .
\end{aligned}
$$

The total energy is reflected toward the inside of the medium. No excited wave is produced. The term "excited" signifies that the energy flux density of the reflected wave is greater than that of the incident one. This case corresponds to that in a linear elastic medium.

$$
\begin{aligned}
& \text { Case [II] } \\
& v_{r 1}=d v_{r 1}, \quad v_{r 2}=v_{s}-d v_{r 2}, \\
& d v_{r 1}=V_{p i}^{1 / 2} v_{s}, \quad d v_{r 2}=0 . \\
& d E_{r x} / d E_{i N} \sim d \theta_{i N} / V_{p i}, \\
& d E_{r z} / d E_{i N} \sim 1 / V_{p i}^{1 / 2} .
\end{aligned}
$$

When $d \theta_{i N}$ is small (near epicenter), the energy ratio $d E_{r x} / d E_{i N}$, the first expression in (6c), is small. As $d \theta_{i N}$ increases, this energy ratio becomes large due to the existence of small $V_{p i}$ in the denominator.

The energy ratio $d E_{r z} / d E_{i N}$, the second expression in (6c), indicates that the excited up-down motion occurs over all range of $d \theta_{i N}$.

The above energy concentration is caused by the slow velocity of the reflected Non-Coupled Simple Wave, i.e., $v_{r 1}=d v_{r 1}$. Similar slow velocity will be frequently found in the Cases [II] and [III] in the subsequent sections.

The above slow velocity occurs as a solution of the truely nonlinear boundary stress condition instead of a linear or quasi-linear one. This point is decisively different from the problem in the linear or quasi-linear case and a very important finding in this paper. The waves due to this small velocity is a clutter near the free surface, most of which is dissipated as a destructive force in the nonlinear case. Some of them is considered to correspond to evanescent factor (exponential part) of Rayleigh-like wave near the free surface in the case of linear theory. Virtually, some solution of small velocity has a possibilty of a trigger to make Rayleigh-type nonlinear wave occur along the free surface, which will be discussed in the later section.

Case [III]

$$
\begin{aligned}
& v_{r 1}=v_{p}+d v_{r 1}, \quad v_{r 2}=d v_{r 2}, \\
& d v_{r 1}=V_{p i} v_{s} /\left(2\left(2+L_{m}\right)^{1 / 2}\right), \\
& d v_{r 2}=d v_{r 2 N} v_{s} /\left(6\left(1+2 L_{m}\right)^{1 / 2}\right. \\
& \left.\quad\left(2+L_{m}\right)^{1 / 2} L_{m}\right) . \\
& d v_{r 2 N}=\mp 3 d \theta_{i N}\left(1+L_{m}\right) \pm 3^{1 / 2} F_{n}^{1 / 2}, \\
& F_{n}=d \theta_{i N}^{2} f_{a 1} \pm f_{a 2} d \theta_{i N} V_{p i}+f_{a 3} V_{p i}^{2}, \\
& f_{a 1}=3\left(1+L_{m}\right)^{2}, \\
& f_{a 2}=16\left(1+2 L_{m}\right)^{3 / 2} L_{m}\left(3+L_{m}\right), \\
& f_{a 3}=2\left(-1+L_{m}\right) L_{m}\left(1+2 L_{m}\right) .
\end{aligned}
$$

By taking significant terms, normalized energy flux density then becomes

$$
\begin{aligned}
& d E_{r x} / d E_{i N} \sim N_{a 1} / V_{p i}+d \theta_{i N} N_{a 2} / V_{p i}, \\
& d E_{r z} / d E_{i N} \sim N_{b 1}+d \theta_{i N} N_{b 2} / V_{p i},
\end{aligned}
$$

where $N_{a 1}, N_{a 2}, N_{b 1}$ and $N_{b 2}$ are functions with respect to $L_{m}$ and $v_{s}$, respectively.

The first term of the energy ratio $d E_{r x} / d E_{i N}$ in (6d) is always large irrespective of $d \theta_{i N}$ due to the existence of only 
small $V_{p i}$ in the denominator. The second term, expressed as $d \theta_{i N} / V_{p i}$, of $d E_{r x} / d E_{i N}$ and $d E_{r z} / d E_{i N}$ in (6d) becomes large as $d \theta_{i N}$ increases, though this ratio is small when $d \theta_{i N}$ is small (near epicenter).

The above concentration of energy near the free surface is caused by the slow velocity of Coupled Simple Wave, i.e., $v_{r 2}=d v_{r 2}$.

\section{Case of Incidence of Coupled Simple Wave \\ 7.1 Incident Coupled Simple Wave}

In the coordinates after the counter-clockwise rotation of the axes $\{x, z\}$ with angle $-\pi / 2+d \theta_{i}$ (see Fig. 1), new axes $\left\{x_{e i C}, x_{f i C}\right\}$ are obtained, where the $x_{e i C}$-axis runs toward the propagation direction of incident Coupled Simple Wave. In Fig. $1, \theta_{i}=\theta_{i C}$, where $\theta_{i C}$ is the incidence angle of incident Coupled Simple Wave. New expressions in the new coordinates are given by the replacements,

$x=x_{e i C}, z=x_{f i C}, u=u_{e i C}, w=u_{f i C}$

in the expression (2d), where $\left\{u_{e i C}, u_{f i C}\right\}$ are the displacement components of the incident Coupled Simple Wave associated with the coordinates $\left\{x_{e i C}, x_{f i C}\right\}$, respectively. That is to say

$$
\begin{gathered}
u_{e i C}=v_{s}^{2} V_{s i}\left(v_{i C} t-x_{e i C}\right) / v_{p}^{2}, \\
u_{f i C}=s_{I} W_{P i}^{1 / 2}\left(v_{i C} t-x_{e i C}\right), \\
W_{P i}=v_{s}^{2} V_{s i}\left(2 v_{p}^{2}-2 v_{s}^{2}-v_{s}^{2} V_{s i}\right) / v_{p}^{4}, \\
V_{s i}=1-v_{i C}^{2} / v_{s}^{2},
\end{gathered}
$$

where $v_{i C}$ and $s_{I}$ are the velocity and the double sign \pm associated with the incident Coupled Simple Wave, respectively.

Let $\left\{u_{i C}, w_{i C}\right\}$ be the displacement components in the $a b$ solute coordinates. These are expressed as

$$
\begin{aligned}
& u_{i C}=S_{i C} u_{e i C}+C_{i C} u_{f i C}, \\
& w_{i C}=-C_{i C} u_{e i C}+S_{i C} u_{f i C}, \\
& S_{i C}=\sin \theta_{i C}, \quad C_{i C}=\cos \theta_{i C} .
\end{aligned}
$$

In the above, the incidence angle $\theta_{i C}$ is a small quantity, since we are treating the case of a direct-hit earthquake, i.e.,

$$
\theta_{i C}=d \theta_{i C} \cdot\left(d \theta_{i C}: \text { a small quantity }\right)
$$

In a procedure similar to that used in the case of the incidence of Non-Coupled Simple Wave (the previous section), we can obtain the energy flux density of reflected waves for each boundary condition [I], [II] and [III] in (1h) in the case of the incidence of Coupled Simple Wave (the present section).

\subsection{Reflected waves in the case of incidence of Coupled Simple Wave}

First of all, the energy flux density of the incident Coupled Simple Wave, $d E_{i C}$, is evaluated.

$$
d E_{i C}=2 v_{s}^{3}\left(v_{p}^{2}-v_{s}^{2}\right) V_{s i} \mu / v_{p}^{4} .
$$

By using the above energy density, the energy flux density of reflected Simple Waves is normalized and the order will be evaluated.

Cases [I], [II] and [III] in the following are the discussions associated with [I], [II] and [III] in boundary conditions (1h).
Case [I]

$$
\begin{aligned}
& v_{r 1}=v_{p}+d v_{r 1}, v_{r 2}=v_{s}-d v_{r 2}, \\
& d v_{r 1}=0, \quad d v_{r 2}=V_{s i} v_{s} / 2 . \\
& d E_{r x} / d E_{i C}=0, \\
& d E_{r z} / d E_{i C}=-1 . \quad(\text { note suffix } z \text { ) }
\end{aligned}
$$

From (8b), the total energy is found to be reflected toward the inside of the medium. No excited wave is generated. This case referrs to that in a linear elastic medium.

Case [II]

$$
\begin{gathered}
v_{r 1}=d v_{r 1}, \quad v_{r 2}=v_{s}-d v_{r 2}, \\
d v_{r 1}=f_{n}^{1 / 2} V_{s i}^{1 / 4}, \quad d v_{r 2}=v_{s} V_{s i} / 2, \\
f_{n}=-s_{I} d \theta_{i C} f_{v}+3 v_{s}^{2} V_{s i}^{1 / 2} / 2, \\
f_{v}=2\left(1+L_{m}\right)^{1 / 2} 2^{1 / 2}\left(3+2 L_{m}\right) \\
v_{s}^{2} /\left(2+L_{m}\right) .
\end{gathered}
$$

By taking the most significant term, the normalized energy flux density becomes

$$
\begin{aligned}
& d E_{r x} / d E_{i C} \sim 1 / V_{s i}^{1 / 2},(\text { note suffix } x) \\
& d E_{r z} / d E_{i C} \sim 0 .
\end{aligned}
$$

Since $V_{s i}$ is small, the above ratio is large, i.e., the excited wave runs along the free surface. This excitation is caused by the retardation of the velocity of the reflected Non-Coupled Simple Wave, i.e., $v_{r 1}=d v_{r 1}$.

Case [III]

$$
\begin{gathered}
v_{r 1}=v_{p}+d v_{r 1}, \quad v_{r 2}=d v_{r 2}, \\
d v_{r 1}=v_{1 r 1} V_{s i}^{1 / 2}, \\
d v_{r 2}=-d \theta_{i N} s_{I} v_{2 r 1} \pm v_{2 r 2} V_{s i}^{1 / 4}, \\
v_{1 r 1}=-3\left(1+2 L_{m}\right)^{1 / 2}\left(1+L_{m}\right)^{1 / 2} v_{s} \\
\qquad /\left(2 \cdot 2^{1 / 2}\left(2+L_{m}\right)^{1 / 2} L_{m}\right), \\
v_{2 r 1}=\left(1+L_{m}\right) v_{s} /\left(2\left(1+2 L_{m}\right)^{1 / 2} L_{m}\right), \\
v_{2 r 2}=f_{r t}^{1 / 2}\left(2+L_{m}\right) v_{s}^{2} /\left(2 L_{m} \mu\right), \\
f_{r t}=4\left(1+2 L_{m}\right)^{1 / 2}\left(1+L_{m}\right)^{1 / 2} 2^{1 / 2} \\
L_{m} \mu^{2} /\left(\left(2+L_{m}\right)^{2} v_{s}^{2}\right) .
\end{gathered}
$$

The significant terms of normalized energy flux density become

$$
\begin{aligned}
& d E_{r x} / d E_{i C} \sim d \theta_{i C} f_{a} / V_{s i}^{1 / 2}+f_{b} / V_{s i}^{1 / 2}, \\
& d E_{r z} / d E_{i C} \sim d \theta_{i C} / V_{s i}^{1 / 2},
\end{aligned}
$$

where $f_{a}$ and $f_{b}$ are the functions with respect to $v_{p}$ and $v_{s}$, respectively.

Since $V_{s i}$ is small, the above ratios become large. Therefore, in the present case, large energy trapping of the reflected wave occurs at the free surface. This excitation of the wave is caused by the retardation of the reflected Coupled Simple Wave, i.e., $v_{r 2}=d v_{r 2}$.

\section{Generation of Rayleigh-type Nonlinear Wave}

In the foregoing section, we obtained the three branches of the boundary conditions [I], [II] and [III] in (1h). In the development of the theory on the occasion of the evaluation of energy flux density, we found that there exist the following 
types of the solutions for the above three branches. That is to say,

$$
\begin{aligned}
& \text { [I] } v_{r 1}=v_{p}+d v_{r 1}, \quad v_{r 2}=v_{s}-d v_{r 2}, \\
& \text { [II } \quad v_{r 1}=d v_{r 1}, \quad v_{r 2}=v_{s}-d v_{r 2}, \\
& \text { [III } \quad v_{r 1}=v_{p}+d v_{r 1}, \quad v_{r 2}=d v_{r 2},
\end{aligned}
$$

where the above [I], [II] and [III] indicate the velocities, $v_{r 1}$ and $v_{r 2}$, of the generated Non-Coupled and Coupled Simple Waves associated with the boundary conditions [I], [II] and [III], respectively.

A Simple Wave (fragment of wave) is a transient wave which changes with the time. After a short time, the incident wave goes out and only the generated waves are left near the free surface.

In this section, let us examine the possibility that the waves lingering near the free surface changes into Rayleigh-type nonlinear wave after the incident Simple Wave ended. The examination will be made for the above three branches of the velocities.

The nonlinear stress-free boundary conditions are given by (1f) with nonlinear stress expressions (1d). Though the form of the stress conditions used in this section is completely the same as that in the foregoing section, the displacement expressions in this section are a little different from those in the foregoing one. In this section, there is no incident wave, so that the displacement expressions are given by

$$
u=u_{r N}+u_{r C}, w=w_{r N}+w_{r C}
$$

instead of (2f). In the above, $\left\{u_{J}, w_{J}\right\}(J=r N, r C)$ are given by $(2 \mathrm{e})$.

In addition to the stress conditions, Snell's law is required along the free surface. That is to say,

$$
v_{r 1} / \sin \theta_{r N}=v_{r 2} / \sin \theta_{r C}=v_{R},
$$

where $v_{R}$ is a supposed velocity of Rayleigh-type wave. The term 'supposed' indicates that such a velocity $v_{R}$ cannot exist in some cases.

The procedure of the analysis is as follows. By using the velocities given in (9a), the displacement expressions (9b), the boundary conditions (1f) and Snell's law (9c), we have the simultaneous equations with respect to $d v_{r 1}$ and $d v_{r 2}$, where first-order terms are retained. From these equations, we can obtain characteristic equation with respect to $v_{R}$. By solving this characteristic equation, the existence of the solution will be examined. The evaluation will be carried out in a weakly nonlinear case where the approximation (1e) is used.

The following Cases [I], [II] and [III] are the discussions associated with [I], [II] and [III] in (9a).

\section{Case [I]}

In this case, the characteristic equation becomes

$$
f_{V}^{2} v_{p}+4 C_{r C} C_{r N} v_{R}^{2} v_{s}^{3}=0,
$$

with

$$
\begin{aligned}
& f_{V}=v_{R}^{2}-2 v_{s}^{2}, \\
& C_{J}=\cos \theta_{J}, \quad(J=r N, r C)
\end{aligned}
$$

As shown in the above, the left-hand side of the equation is always positive. Therefore, there exists no solution $v_{R}$ satisfying the equation.

Case [II]

In this case, the characteristic equation becomes

$$
\left(1+\left(2 d v_{r 2} v_{s}\right) / v_{R}^{2}-v_{s}^{2} / v_{R}^{2}\right)^{1 / 2}=0 .
$$

Solving the above equation, we have

$$
v_{R}=v_{s}-d v_{r 2} . \quad\left(d v_{r 2}>0\right)
$$

As found in the above, Rayleigh-type nonlinear wave appear along the free surface. The propagation velocity is then a little smaller than that of linear $S$ wave.

The ratio of energy flux density, $d E_{r x} / d E_{r z}$, is evaluated, where $d E_{r x}$ and $d E_{r z}$ stand for the energy flux density (3c) of the total displacement components (9b) in the direction of the $x$ - and $z$-axes, respectively. The evaluated result is

$$
d E_{r x} / d E_{r z} \sim 1 / d v_{r 1}
$$

The above ratio is very large, since $d v_{r 1}$ is a small perturbation term. That is to say, most of energy is directed along the free surface.

\section{Case [III]}

In this case, the characteristic equation becomes

$$
\begin{aligned}
& 1 \pm f_{c 1} f_{r t}^{1 / 2}+f_{c 0} v_{R}^{2}=0, \\
& f_{r t}=2 d v_{r 1 r} v_{p}-v_{p}^{2}+v_{R}^{2}, \\
& f_{c 0}=-\left(2+L_{m}\right) /\left(2 v_{s}^{2}\right), \\
& f_{c 1}=\left(1+2 L_{m}\right)^{1 / 2} /\left(\left(2+L_{m}\right)^{1 / 2} v_{s}\right) .
\end{aligned}
$$

By solving the above characteristic equation, it is found that $v_{R}$ has only complex (not real) solution. Therefore, Rayleigh-type nonlinear wave does not occur in this case.

Through the above evaluation in Cases [I] to [III], only the retardation of nonlinear $P$ wave (Case [II], then $v_{r 1}=d v_{r 1}$ ) produces Rayleigh-type nonlinear wave. It must be here noted that this generation of Rayleigh-type nonlinear wave occurs in the case of truely nonlinear stress surface condition instead of quasi-linear one (in such cases as Taylor expansion of stress expression by small parameter, as treated by the authors cited in the introduction).

Some mention is made here on the discussion in this section. Though we gave three branches of velocity components [I] to [III] in the beginning of this section, these branches are obtained from the concept such that the incident waves are incident on the free surface at a small incidence angle, i.e., in the case of direct-hit earthquake. Therefore, unknown type of the velocity other than the above-mentined three branches may be expected in the surface range apart from epicenter or the outside of direct-hit range.

\section{Summary}

The basic concept of the analysis is based on the fact such that nonlinear waves can be expressed as the combination of several fragments of Simple Waves (previous study). In the 
present study, the incident and reflected nonlinear waves are, therefore, given by Simple Waves.

In a nonlinear elastic medium, the expression of stress is of nonlinear form with respect to displacement. In due course, the expression of stress condition at a free surface becomes nonlinear with respect to displacement in a model of half-infinite elastic medium.

On the occasion of the incidence of nonlinear waves on the free surface, several branches of reflected waves are generated by nonlinear stress condition.

In this paper, the treatment is based on second order nonlinear stress and further limited to the case of a direct-hit earthquake. The restriction of 'direct-hit' signifies small incidence angle of the incident waves upon the free surface. In addition to the above condition, weak nonlinearity of the medium is assumed, where elastic coefficients associated with third order terms are negligibly small in energy function. This procedure make easy the physical interpretation of the results.

Under these conditions, the surface condition is separated into three cases (branches). The first one is a condition which generates reflected waves with a velocity near that of $P$ or $S$ wave in the linear theory. The other two are conditions which usually generate retarded waves with a slow velocity. These retarded waves are the most important finding in this study. The last-mentioned retarded waves produce high energy flux along the free surface. This feature causes large disaster at the free surface on the occasion of large earthquake. Most of energy is then dissipated as energy of destruction near the free surface.

Rayleigh-type nonlinear wave appears along the free surface. The propagation velocity along the free surface is then a little slower than that of linear $S$ wave. The above Rayleigh wave is produced only in the case where nonlinear $P$ wave is retarded along the free surface.

It is noted that the above findings of the slow velocity and Rayleigh-type nonlinear wave are obtained by use of truely nonlinear stress condition at the free surface instead of quasilinear one based on perturbation procedure.

\section{References}

Blant, D. R., Nonlinear Dynamic Elasticity, 93 pp., Blaisdell publishing Company, England, 1969.

Engelbrecht, J., Nonlinear Wave Processes of Deformation in Solids, 223 pp., Pitman, London, 1983.

Engelbrecht, J., Nonlinear Wave Dynamics: Complexity and Simplicity, 183 pp., Kluwer Academic Publishers, 1997.

Engelbrecht, J., V. E. Fridman, and E. N. Pelinovski, Nonlinear Evolution Equations, 122 pp., Longman, Harlow, 1988.

Hazewinkel, M., H. W. Capel, and E. M. de Jager, Proceedings of the International Symposium, held in Amsterdam, The Netherlands, 23-26 April 1995, 516 pp., Kluwer Academic Publishers, 1995.

Jeffrey, A. and J. Engelbrecht, Nonlinear Waves in Solids, 382 pp., SpringerVerlag, Wien-New York, 1994.

Jeffrey, A. and T. Kawahara, Asymptotic Methods in Nonlinear Wave Theory, 256 pp., Pitman, London, 1982.

Litvin, A. L. and I. D. Tsvankin, Interaction of plane waves with the boundary of a nonlinear elastic medium, in Problems of Nonlinear Seismology, edited by A. N. Nikolaev and I. N. Galkin, Nauka, Moskow, pp. 128-136, 1987 (in Russian).

Momoi, T., Wave propagation in nonlinear-elastic isotropic media, Bull. Earthq. Res. Inst., 65, 413-432, 1990.

Momoi, T., The polarization of waves in an anisotropic nonlinear-elastic medium, Bull. Earthq. Res. Inst., 67, 1-20, 1992.

Momoi, T., Simple Waves characterizing wave propagation in a nonlinear elastic medium, Earth Planets Space, 51, 315-319, 1999.

Parker, D. F. and F. M. Talbot, Nonlinear elastic surface waves, in Nonlinear Deformation Waves, edited by U. Nigul and J. Engelbrecht, pp. 397-403, Springer, Berlin-Heidelberg, 1983.

Taniuti, T. and K. Nishihara, Nonlinear Waves, 258 pp., Pitman, London, 1977.

T. Momoi (e-mail: qyz1932@pb.highway.ne.jp) 